\section{Case Reports in Ophthalmology}

Case Rep Ophthalmol 2020;11:620-625

\title{
Twelve-Year-Old Girl with Acute Macular Neuroretinopathy: A Multimodal Retinal Evaluation
}

\author{
Luis Filipe Nakayama Camila Kase Vinicius Campos Bergamo \\ Nilva Simeren Bueno de Moraes \\ Physician Department of Ophthalmology, Universidade Federal de São Paulo - EPM, \\ São Paulo, Brazil
}

\section{Keywords}

Retina $\cdot$ Multimodal retinal exams · Optical coherence tomography angiography

\section{Abstract}

We report a 12-year-old female patient with acute macular neuroretinopathy assessed with multimodal retinal exams. Initial fluorescein angiogram and optical coherence tomography angiography (OCT-A) were both normal, and after 4 months OCT-A showed abnormal vascular flow with normal flow in superficial retina layers, choroid, and choriocapillaris.

\section{Background}

Acute macular neuroretinopathy (AMN) is a rare disease, more common in young female patients [1]. AMN was first described in 1975 by Bos and Deutman [2] as intraretinal dark reddish wedge-shaped lesions. The exact pathophysiology is yet unknown, and the proposed mechanism is ischemia in deep retinal capillary plexus or in choriocapillaris, but this does not fully explain optical coherence tomography (OCT) findings [3].

OCT angiography (OCT-A) is a noninvasive exam that evaluates retinal and choroid vascular plexus through optic interferometry [4]. 


\section{Case Reports in Ophthalmology}

Case Rep Ophthalmol 2020;11:620-625 DOI: 10.1159/000509849

(c) 2020 The Author(s). Published by S. Karger AG, Basel www.karger.com/cop

Nakayama et al.: Twelve-Year-Old Girl with Acute Macular Neuroretinopathy: A Multimodal Retinal Evaluation

We describe a 12-year-old female patient with clinical and OCT compatible AMN findings, who presented an initial normal OCT-A, and after 4 months, showed a flow deficit in deep retinal vascular layers.

\section{Case Report}

A 12-year-old female patient with a chief complaint of sudden visual scotoma in right eye. She denied past ocular history or flu-like symptoms, and only reported use of combined oral contraceptive pill (drospirenone $3 \mathrm{mg}$ and ethinyl estradiol $0.03 \mathrm{mg}$ ) due to polycystic ovary syndrome for 5 months.

At ophthalmological exam, she had a visual acuity of 20/20 ( -1.0 spherical diopters) in both eyes. Normal anterior biomicroscopy intraocular pressure of $14 \mathrm{~mm} \mathrm{Hg}$ in both eyes and the pupillary exam was normal. She presented abnormal retinal reflex in the macular area of the left eye.

Color fundus retinography showed subtle abnormal retina in the macular area of the right eye, more visible on infra-red retinography. Red-free were normal, and autofluorescence imaging showed a subtle hypo-signal in the macular area (Fig. 1).

Fluorescein angiogram was normal in all phases in both eyes, without hyper- or hypofluorescence areas during the exam (Fig. 1).

OCT showed areas of hyper-signal in deep retina, corresponding to areas from infra-red retinography (Fig. 2).

Initial OCT-A did not show areas of flow deficit in the superficial retina, deep retina, choriocapillaris, and choroid (Fig. 3).

Four months after onset of symptoms, the patient reported improvement of visual scotoma, the OCT showed thinning of retinal layers (Fig. 3), and OCT-A showed a decrease in vascular flow in an area corresponding to AMN. Vascular decrease flow was present in deep retinal vascular layer. Superficial retinal layer and choriocapillaris were both normal in the initial and the follow-up exam (Fig. 4).

\section{Discussion}

We report a case of acute macular neuroretinopathy in a 12-year-old female patient, one of the youngest AMN patients reported in the literature.

This patient did not have any past ocular history and started taking combined oral contraceptive pills 5 months ago. Although combined oral contraceptive pill use is associated with AMN, it is difficult to establish a direct correlation between its use and AMN [1].

Krill disease is a possible differential diagnosis, but OCT lesion location does not correspond with that disease. White dots syndromes such as multiple evanescent white dot syndrome and acute posterior multifocal placoid pigment epitheliopathy and central serous chorioretinopathy are possible differential diagnoses, but ophthalmological findings and ancillary retinal exams favored the diagnosis of AMN.

Exact AMN pathophysiology is yet unknown, and previous reports with OCT-A speculated a choroidal disease with retinal involvement [3]. Our case reports an initial normal vasculature in all retinal and choroid layer in on OCT-A, with flow deficit mainly at the deep retinal plexus layer at the 4-month follow-up OCT-A. These findings contribute to the hypothesis that AMN is a deep vascular plexus disease. In OCT-A, shadowing artifacts may occur on images, 
but the fundus color retinography did not show any hemorrhage or opacity that could have prevented light from reaching the tissue. To avoid segmentation artifact, the exam was manually corrected for the previous automatic segmentation in all slabs.

With a normal retinal fundus exam, multimodal retinal exams helped to establish AMN diagnosis and follow-up, mainly infrared retinography, OCT, and OCT-A.

In this disease, OCT-A is a good option for diagnosis and follow-up, since capillary plexus ischemia usually is not significant enough to change fluorescein angiogram exam.

\section{Conclusion}

AMN is a rare disorder, and multimodal exams help to better understand the characteristics of this disease. This is one of the youngest patients with an AMN reported to date.

\section{Acknowledgements}

We would like to thank Ophthalmology sector from Escola Paulista de Medicina/São Paulo Federal University.

\section{Statement of Ethics}

Ethics approval is not applicable. Informed consent for publication of clinical details and images was obtained from the patient.

\section{Conflict of Interest Statement}

No competing interests to declare.

\section{Funding Sources}

No funding to declare.

\section{Author Contributions}

L.F. Nakayama: project concept, examination, article writing, review. V.C. Bergamo: article writing, review. N.S.B. Moraes: project concept, review, project coordinator. All authors read and approved the final manuscript.

\section{Availability of Data and Material}

The datasets generated during the current study are available upon reasonable request from the corresponding author L.F. Nakayama. 


\section{Case Reports in Ophthalmology}

\begin{tabular}{l|l}
\hline DOI: $10.1159 / 000509849$ & (c) 2020 The Author(s). Published by S. Karger AG, Basel \\
www.karger.com/cop
\end{tabular}
www.karger.com/cop

Nakayama et al: Twelve-Year-Old Girl with Acute Macular Neuroretinopathy: A Multimodal Retinal Evaluation

\section{References}

1 Bhavsar KV, Lin S, Rahimy E, Joseph A, Freund KB, Sarraf D, et al. Acute macular neuroretinopathy: A comprehensive review of the literature. Surv Ophthalmol. 2016 Sep-Oct;61(5):538-65.

2 Bos PJ, Deutman AF. Acute macular neuroretinopathy. Am J Ophthalmol. 1975 Oct;80(4):573-84.

3 Thanos A, Faia LJ, Yonekawa Y, Randhawa S. Optical Coherence Tomographic Angiography in Acute Macular Neuroretinopathy. JAMA Ophthalmol. 2016 Nov;134(11):1310-4.

4 Kashani AH, Chen CL, Gahm JK, Zheng F, Richter GM, Rosenfeld PJ, et al. Optical coherence tomography angiography: A comprehensive review of current methods and clinical applications. Prog Retin Eye Res. 2017 Sep;60:66-100.

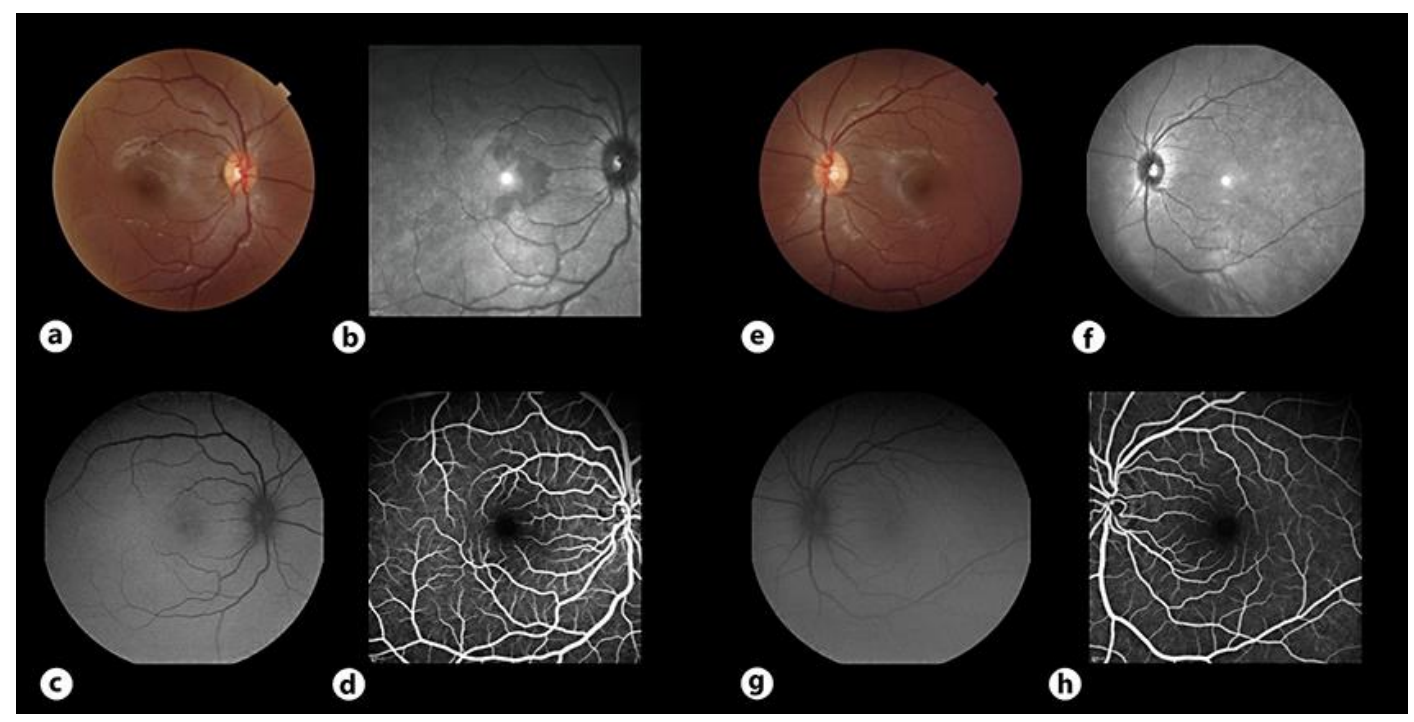

Fig. 1. Imaging on presentation. a Right eye fundus photography without visible changes. b Right eye near infrared fundus fluorescence with circular petaloid lesion nasal to the fovea. c Right eye normal fundus autofluorescence. $\mathbf{d}$ Right eye normal intravenous fluorescein angiography. e Left eye fundus photography without visible changes. $\mathbf{f}$ Left eye normal near infrared fundus fluorescence. $g$ Left eye normal fundus autofluorescence. $\boldsymbol{h}$ Left eye normal intravenous fluorescein angiography. 

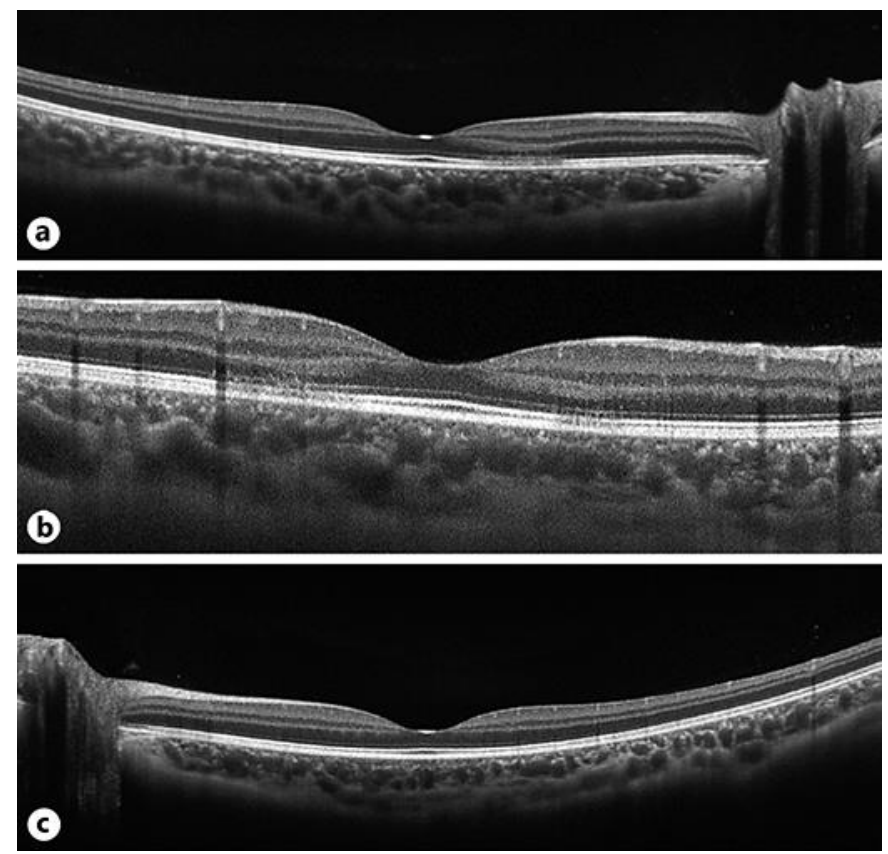

Fig. 2. Swept source OCT. a Right eye horizontal slab with increased reflectiveness in intermediate layers nasal to the fovea. b Right eye vertical slab with increased reflectiveness in intermediate layers superior and inferior to the fovea. c Left eye horizontal slab with normal retinal layers on OCT.

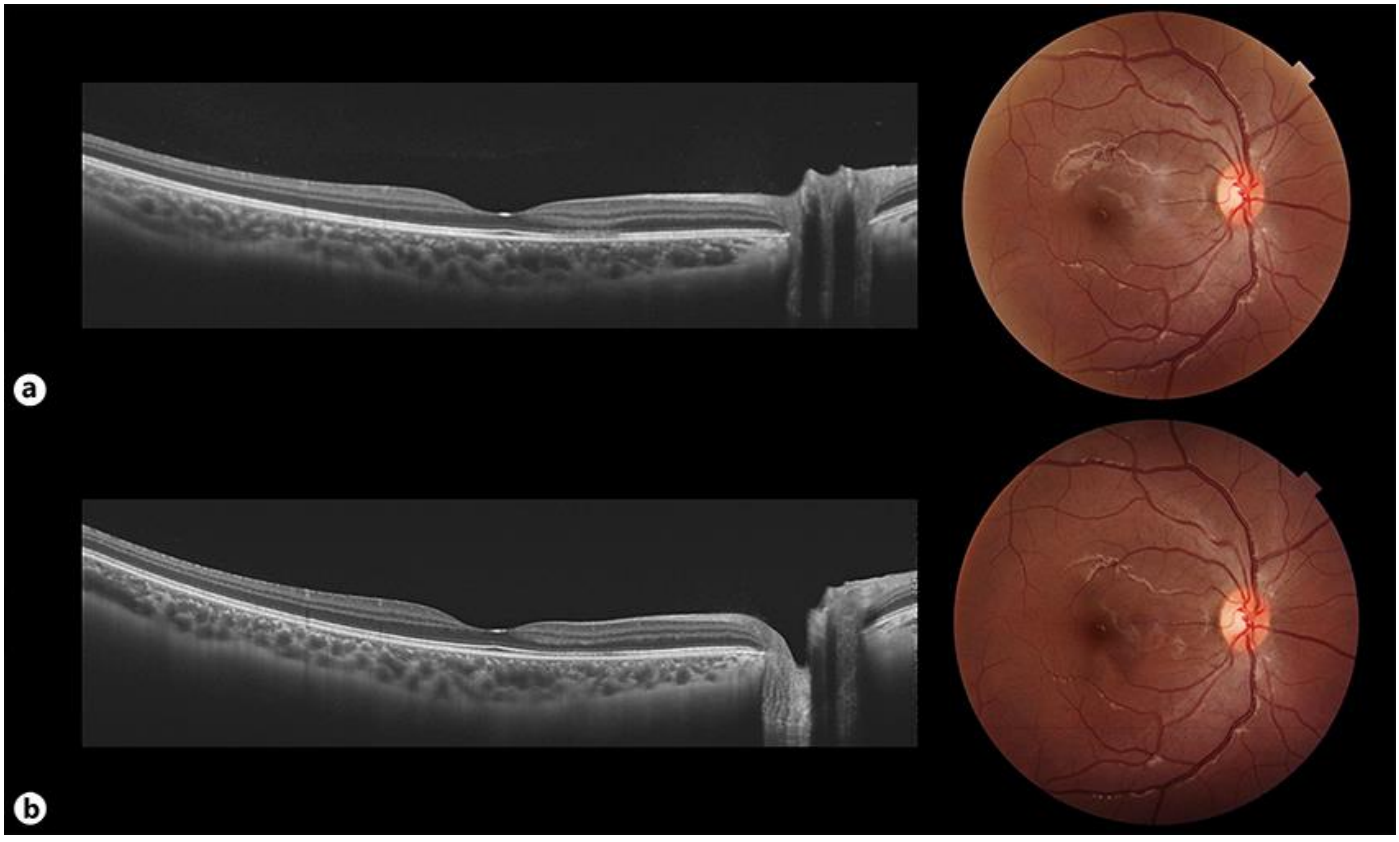

Fig. 3. a Initial presentation. OCT with increased reflectiveness in intermediate layers nasal to the fovea. b Four months' follow-up. Increased retinal thickness and irregularity in intermediate retinal layers. Improvement of hyperreflectiveness in intermediate retinal layers. 


\section{Case Reports in Ophthalmology}

Nakayama et al.: Twelve-Year-Old Girl with Acute Macular Neuroretinopathy: A Multimodal Retinal Evaluation
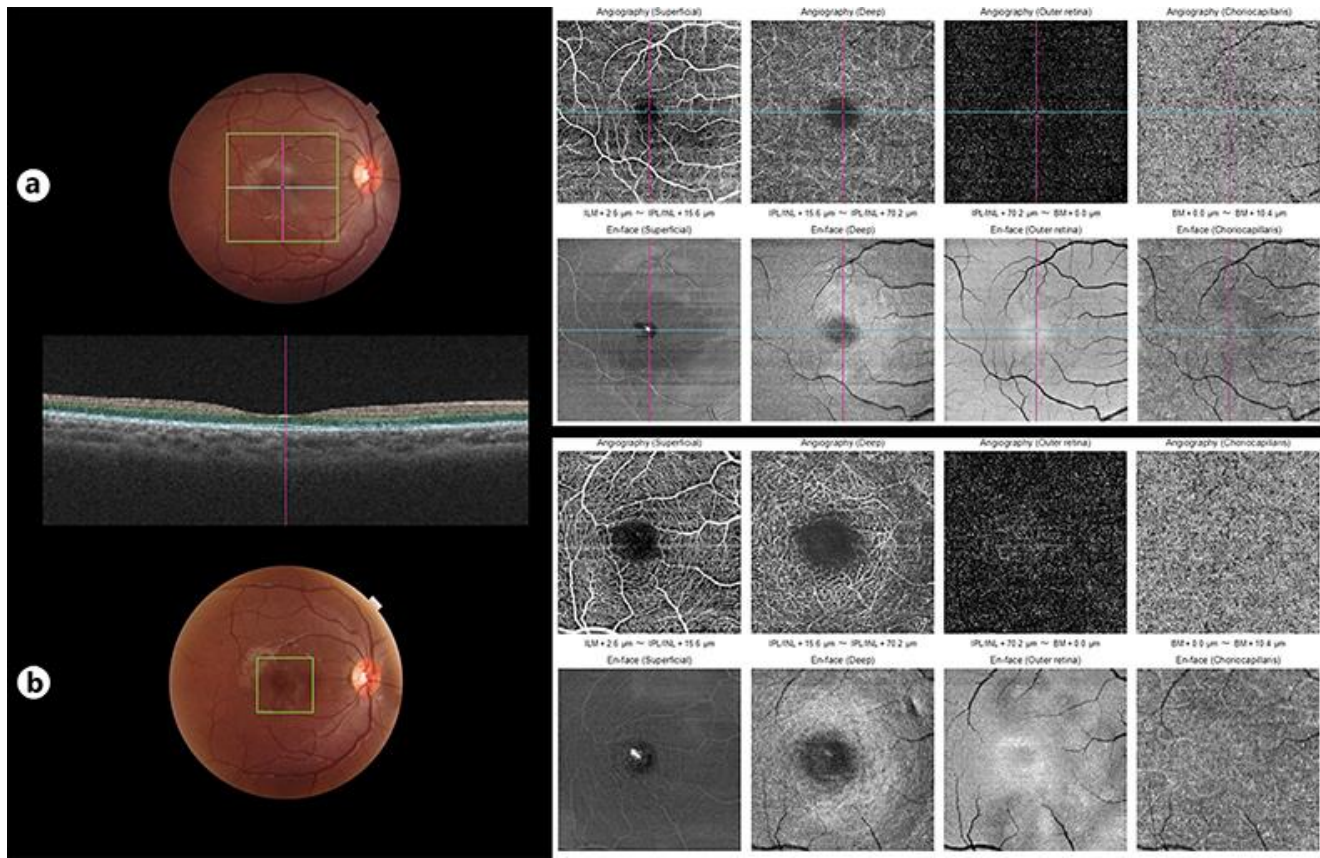

Fig. 4. OCT-A of right eye. a Imaging on presentation. OCT-A in all layers with normal vasculature. Crescent-shape lesion visible in outer retina en-face exam. b Four months' follow-up. Decreased flow in deep retina plexus in corresponding area to near infra-red lesion. Normal superficial, outer retina and choriocapillaris plexus. Crescent-shape lesion visible in deep and outer retina en-face exam. 